\title{
Protein AATF
}

National Cancer Institute

\section{Source}

National Cancer Institute. Protein AATF. NCI Thesaurus. Code C92523.

Protein AATF (560 aa, $\sim 63 \mathrm{kDa}$ ) is encoded by the human AATF gene. This protein may play a role in apoptosis and transcription. 